\title{
Challenges and Critical Water Quality Issues in Micro Water Systems in British Columbia, Canada
}

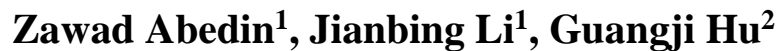 \\ ${ }^{1}$ School of Engineering, University of Northern British Columbia \\ 3333 University Way, Prince George, BC, Canada \\ abedin@unbc.ca; jianbing.li@unbc.ca \\ ${ }^{2}$ School of Engineering, University of British Columbia \\ 3333 University Way, Kelowna, BC, Canada \\ guangji.hu@ubc.ca
}

\section{Extended Abstract}

One-sixth of the world population suffers from the lack of clean water supply [1]. Water insecurity is defined as inadequate or inequitable access to clean, safe and affordable water for drinking, cooking, as well as sanitation and hygiene [2]. In Canada, the National Assessment of First Nations Water and Wastewater Systems (2011) shows that 73\% of water systems were at medium to high levels of risk because the facilities could not meet the design protocols or substandard water quality [3]. In British Columbia (BC), small systems are defined as the water system that serves 500 or less people. A water system that serves 25 or less people (less than 5 household/connection) is called a microsystem.

As of January 2021, 57 long-term drinking water advisories were still in effect in 39 water systems with 5 or more connections in First Nations communities [4]. As per the data accessed in February 2021[5,6], Fraser Health region and Northern health region have 9 and 126 different types of ongoing drinking water advisories, respectively, such as Boil Water Advisory (BWA), Do Not Consume (DNC), Water Quality Advisory (WQA). About 22\% of those advisories are due to coliforms, which can be solved easily with common disinfection processes (e.g., hypochlorite solution), and $61 \%$ of advisories are due to lack of assessment data. Water quality assessments commonly involve the evaluation of water quality data, in terms of different parameters according to water quality guidelines and standards. All of these specifics clearly indicate the need of drinking water quality data acquisition and analysis, and especially for micro-systems since those systems are out of funding and assistance from government.

Water quality data of microsystems from participating communities were acquired and analyzed using correlation and principal component analysis. The results show that the most critical water quality issue is related to Manganese (Mn) in drinking water. Since BWA advisory is the most common advisory to circulate for making people aware of potential water contamination, boiling makes Mn contamination worse; DNC advisory is issued in case of such contamination. The results will help to further study and develop a fit-for-purpose solution which needs to be cost-effective as well, to help residents relying on microsystems to deal with Mn problem specifically. Therefore, such solution can help address similar Mn contamination problems in other regions across the globe as well.

\section{References}

[1] M. Emelech, "The global challenge for adequate and safe water," Journal of Water Supply: Research and TechnologyAqua, vol. 55, no. 1, p. 3-10, 2006.

[2] C. C. Schimpf, "A Systematic Literature Review on Water Insecurity from an Oregon Public Health Perspective," International Journal of Environmental Research and Public Health, vol. 17, no. 3, pp. 1-23, 2020.

[3] Department of Indian and Northern Affairs Canada, "National Assessment of First Nations Water and Wastewater Systems," Department of Indian and Northern Affairs Canada, Orangeville, Ontario, 2011.

[4] Indigenous Services Canada (2021, Jan 10). Ending long-term drinking water advisories [Online]. Available: https://www.sac-isc.gc.ca/eng/1506514143353/1533317130660

[5] Fraser Health (2021, Feb 21). Drinking water advisories [Online]. Available: https://www.healthspace.ca/Clients/FHA/FHA_Website.nsf/Water-Drinking-Closures

[6] Northern Health (2021, Feb 21). Drinking water advisories [Online]. Available: https://www.northernhealth.ca/services/environmental-health/drinking-water/advisories-and-notifications 